\title{
Spinal Position Sense and Trunk Muscle Activity During Sitting and Standing in Nonspecific Chronic Low Back Pain
}

\author{
Classification Analysis \\ Liba Sheeran, $\mathrm{PhD},{ }^{*}$ Valerie Sparkes, PhD, * Bruce Caterson, PhD, † Monica Busse-Morris, PhD, ${ }^{*}$ and \\ Robert van Deursen, $\mathrm{PhD}^{*}$
}

Study Design. A cross-sectional study between subgroups of nonspecific chronic low back pain (NSCLBP) and asymptomatic controls.

Objective. To investigate NSCLBP subgroup differences in spinal position sense and trunk muscle activity when repositioning thoracic and lumbar spine into neutral (midrange) spinal position during sitting and standing.

Summary of Background Data. Patients with NSCLBP report aggravation of symptoms during sitting and standing. Impaired motor control in NSCLBP, associated with sitting and standing postures nearer the end range of spinal motion, may be a contributing factor. Rehabilitation improving neutral (midrange) spinal position control is advocated. Postural and motor control alterations vary in different NSCLBP subgroups, potentially requiring specific postural interventions. There is limited evidence on whether subgroup differences exist when performing neutral spine position tasks.

Methods. Ninety patients with NSCLBP and 35 asymptomatic controls were recruited. Two blinded practitioners classified NSCLBP into subgroups of active extension pattern and flexion pattern. Participants were assisted into neutral spine position and asked to reproduce this position 4 times. Absolute, variable, and constant errors were calculated. Three-dimensional thoracic and lumbar kinematics quantified the repositioning accuracy and

From the *Research Centre for Clinical Kinaesiology, School of Healthcare Studies, Cardiff University, Cardiff, Wales, UK; and +School of Biosciences, Cardiff University, Cardiff, Wales, UK.

Acknowledgment date: May 19, 2011. First revision date: August 18, 2011. Acceptance date: September 3, 2011.

The manuscript submitted does not contain information about medical device(s)/drug(s).

Corporate/industry funds were received to support this work. Although one or more of the author(s) has/have received or will receive benefits for personal or professional use from a commercial party related directly or indirectly to the subject of this manuscript, benefits will be directed solely to a research fund, foundation, educational institution, or other nonprofit organization which the author(s) has/have been associated.

Address correspondence and reprint requests to Liba Sheeran, PhD, Research Centre for Clinical Kinaesiology, School of Healthcare Studies, Cardiff University, Ty Dewi Sant, Heath Park, Cardiff CF14 4XN, Wales, UK; E-mail: sheeranL@cardiff.ac.uk

DOI: 10.1097/BRS.0b013e31823b00ce surface electromyography assessed back and abdominal muscles activity bilaterally.

Results. Irrespective of subclassification, patients with NSCLBP produced significantly greater error magnitude and variability than the asymptomatic controls, but subgroup differences were detected in the error direction. Subgroup differences in the trunk muscle activity were not consistently identified. Although both subgroups produced significantly higher abdominal activity, subclassification revealed difference in superficial multifidus activity during standing, with flexion pattern producing significantly greater activity than the asymptomatic controls.

Conclusion. Subgroups of NSCLBP had similar neutral spinal position deficits regarding error magnitude and variability, but subclassification revealed clear subgroup differences in the direction of the deficit. The trunk muscle activation was shown to be largely nondiscriminatory between subgroups, with the exception of superficial lumbar multifidus.

Key words: nonspecific chronic low back pain, subclassification, thoracic and lumbar repositioning sense, trunk muscle activity.

Spine 2012;37:E486-E495

ow-back pain (LBP) is a highly prevalent and costly musculoskeletal pain syndrome, ${ }^{1,2}$ associated with recurrence and chronicity. ${ }^{3}$ Approximately $75 \%$ to $85 \%$ of cases are classified as nonspecific chronic low back pain (NSCLBP), with no detected evidence of pathoanatomical/ radiological abnormality. ${ }^{4}$

From clinical observations, impaired motor control during sitting and standing, potentially leading to changes in spinal posture, has been proposed to contribute to LBP. ${ }^{5,6}$ The evidence is inconsistent; some studies report sitting and standing to increase the risk for $\mathrm{LBP}^{7-10}$ whereas other studies show no relationship. ${ }^{11,12}$ Moreover, a recent systematic review revealed limited evidence for a causal link between spinal postures and back pain. ${ }^{13}$ The reviewed studies were of poor methodological quality, however, ${ }^{13}$ and included largely heterogeneous LBP samples, potentially contributing to a large variation in spinal postures and difficulty identifying differences compared with healthy population. 
Classifying NSCLBP on the basis of pain-provoking postures and movements, using a classification system developed by O'Sullivan, ${ }^{6,14}$ revealed that compared with healthy controls, CLBP patients have altered spinal postures that vary between the subgroups ${ }^{15,16}$ and are associated with subgroup-specific trunk muscle activity changes. ${ }^{17}$ Specific CLBP populations also demonstrated reduced ability to adopt and/or maintain a neutral (midrange) position of the spine ${ }^{18}$ and a tendency to adopt postures nearer the end range of spinal motion compared with controls. ${ }^{5,10,19,20}$ Although the causal relationship is unclear, long-term exposure of the spinal viscoelastic tissues to sustained or cyclic mechanical loads of high frequencies and/or rates has been shown to trigger acute inflammatory response indicative of collagen microdamage ${ }^{21,22}$ and neuromuscular dysfunction, ${ }^{23,24}$ suggested to lead to chronic inflammation and cumulative back pain disorder. ${ }^{25}$

Postural rehabilitation to improve neutral (midrange) position awareness and control forms the basis of LBP management in clinical practice. ${ }^{26,27}$ The NSCLBP subgroup variation in postures, however, led to recommendations that rehabilitation must reflect subgroup variation and must be matched to the specific impairment. ${ }^{16,17,28}$

To develop subgroup-specific postural rehabilitation, it is important to determine whether differences exist in how subgroups perform neutral spine position tasks. Adequate performance of posture tasks depends on accurate sensory and motor function. ${ }^{29}$ Some evidence exists that individuals with LBP have reduced neutral spine position sense during sitting, ${ }^{18,30}$ with an indication of a directional specificity in flexion-related LBP. ${ }^{18}$ However, sensory and motor function during neutral spine positions has not yet been investigated in different positions, spinal regions, and other NSCLBP subgroups.

The aim of the study was to investigate whether thoracic and lumbar neutral spine position sense and trunk muscle activity differences exist during sitting and standing in NSCLBP when considered as a whole and subclassified. ${ }^{31}$

\section{MATERIALS AND METHODS}

Of 224 patients with CLBP, referred for physiotherapy at Cardiff and Vale University Health Board, Wales (UK), 92 met the selection criteria (Table 1) and consented to participate. Asymptomatic controls ( $\mathrm{n}=38 ; 22$ female participants), matched for sex and age; no LBP (minimum $1 \mathrm{yr}$ ); and no vestibular, visual, and neurological condition affecting balance, were recruited. The South East Wales Research Ethics Committee and the Cardiff and Vale University Health Board Research Committee approved the study. Sample size was based on a power calculation of repositioning error data published previously. ${ }^{32} \mathrm{~A}$ mean difference of $1.79^{\circ}$ (common standard deviation of 2.68$)^{32}$ gave an effect size of 0.66 . Assuming a power of $80 \%$ and alpha level of 0.05 , this equated to a sample of 38 subjects per group. ${ }^{33}$

Modified visual analogue scale, ${ }^{34}$ evaluating pain "on average," "at its worst and best" and accepted to be representative of CLBP, ${ }^{34}$ assessed pain. The Roland-Morris Disability Questionnaire, ${ }^{35}$ which is reliable in $\mathrm{CLBP}^{36}$ and
TABLE 1. Inclusion and Exclusion Criteria for the Nonspecific Chronic Low Back Pain (NSCLBP) Group*

Inclusion criteria

LBP for a minimum of 12 weeks

Pain in the lumbar and buttock regions

Clear mechanical basis of disorder: specific postures and movements that aggravate and ease the symptoms, and symptom relief in movements opposite to provocation testing as determined by subjective and objective clinical examination

Clinical diagnosis of FP or AEP motor control impairment

Key clinical features of FP

Symptoms provoked with movements and postures involving flexion of lower lumbar spine

Symptoms eased by movements into spinal extension

Loss of segmental lordosis, difficulty of adopting and/or maintaining neutral spine posture, with tendency toward flexed lower lumbar spine

Key clinical features of AEP

Symptoms provoked with movements and postures involving extension of lower lumbar spine

Symptoms eased by movements into spinal flexion

Difficulty of adopting and/or maintaining neutral spine posture, with tendency toward hyperextension of lower lumbar spine

Exclusion criteria

Not fulfilling inclusion criteria

Red flags (specific causes of LBP, such as disc prolapse with radicular pain, inflammatory disease, or other serious pathology)

Dominant yellow flags (evidence of distress on Distress Risk Assessment Method) ${ }^{31}$

Pregnancy/breastfeeding

History of spinal surgery

Vestibular/visual/neurological dysfunction affecting balance

Not able to sit and/or stand from a stool unaided

*All inclusion criteria had to be satisfied based on O'Sullivan. ${ }^{6,14}$

LBP indicates low back pain; FP, flexion pattern; $A E P$, active extension pattern.

correlates with other disability questionnaires, ${ }^{37,38}$ assessed disability.

\section{SUBCLASSIFICATION}

A validated O'Sullivan's classification system ${ }^{6,14,39}$ was used to subclassify patients using a process of diagnostics described elsewhere. ${ }^{40}$ It includes (1) subjective assessment of the disorder history, aggravating and easing activities, and (2) functional movement and accessory/physiological joint assessment 


\begin{tabular}{|c|c|c|c|c|}
\hline \multicolumn{5}{|c|}{$\begin{array}{l}\text { TABLE 2. Group Characteristics for the } \\
\text { Asymptomatic Controls and } \\
\text { Nonspecific Chronic Low Back Pain } \\
\text { Patients With the FP and the AEP }\end{array}$} \\
\hline & $\begin{array}{l}\text { Controls } \\
(\mathrm{n}=35)\end{array}$ & $\begin{array}{c}\text { FP } \\
(n=51)\end{array}$ & $\begin{array}{c}\text { AEP } \\
(n=39)\end{array}$ & $\begin{array}{c}\text { Test } \\
\text { Statistic }\end{array}$ \\
\hline \multicolumn{5}{|l|}{$\operatorname{Sex}(\%)$} \\
\hline Men & $13(37.1 \%)$ & $22(43.1 \%)$ & $9(23.1 \%)$ & $P=0.139$ \\
\hline Women & $22(62.9 \%)$ & $29(56.9 \%)$ & $30(76.9 \%)$ & \\
\hline $\begin{array}{l}\text { Age (SD) } \\
(\mathrm{yr})\end{array}$ & $36.0(10.3)$ & $33.0(10.3)$ & $37.0(11.4)$ & $P=0.316$ \\
\hline $\begin{array}{c}\mathrm{BMI}(\mathrm{SD}) \\
\left(\mathrm{kg} / \mathrm{m}^{2}\right)\end{array}$ & $23.3(2.2)$ & 25.1 (3.6) & $24.9(3.8)$ & $\begin{array}{l}P=0.03^{*} \\
\text { FP vs. } \mathrm{H}^{*} \\
\text { AEP vs. H } \\
\text { AEP vs. FP }\end{array}$ \\
\hline VAS (SD) & $\ldots$ & $4.8(1.3)$ & $4.5(1.4)$ & $P=0.306$ \\
\hline $\begin{array}{l}\text { RMDQ } \\
\text { (SD) }\end{array}$ & $\ldots$ & $7.3(3.8)$ & $6.2(3.5)$ & $P=0.177$ \\
\hline \multicolumn{5}{|c|}{$\begin{array}{l}{ }^{*} \text { Significant at } P<0.05 . \\
F P \text { indicates flexion pattern; } A E P \text {, active extension pattern; BMI, body mass } \\
\text { index; } H \text {, healthy; VAS, visual analogue scale; RMDQ, Roland-Morris Dis- } \\
\text { ability Questionnaire. }\end{array}$} \\
\hline
\end{tabular}

to determine the type and level of the impairment. ${ }^{6,41}$ The functional movement assessment (forward/backward bending, sitting, standing, sit-to-stand, and a single leg stance) was video-recorded with 2 camcorders (posterior and sagittal views). Pain behavior and video data were used by 2 experienced physiotherapists (LS, VS) to subclassify. An identical classification process has been used previously, ${ }^{15-17,42}$ demonstrating good interexaminer reliability. ${ }^{42}$ Patients with clinical signs of a flexion pattern (FP) and an active extension pattern (AEP) (Table 1) were selected.

\section{EXPERIMENTAL PROTOCOL}

Testing was performed at the Research Centre for Clinical Kinaesiology, Cardiff University. Spinal position sense and trunk muscle activity were evaluated during participants' attempts to reproduce a target position of neutral lumbar lordosis and neutral thoracic kyphosis. The neutral spine position was defined as a thoracic and lumbar midrange position between end-range flexion and extension. Participants were blindfolded and wore loose clothing to minimize sensory cues.

Each participant was first seated on a fixed-height stool, ${ }^{30}$ with feet positioned shoulder width apart and arms placed loosely on thighs. Participants were assisted in moving through their available range of spinal flexion and extension 3 times. They were then positioned by the researcher (LS) into a thoracic and lumbar neutral (midrange) position for 5 seconds and instructed to memorize it because they would be asked to reproduce it during the test trials. ${ }^{18}$ Participants

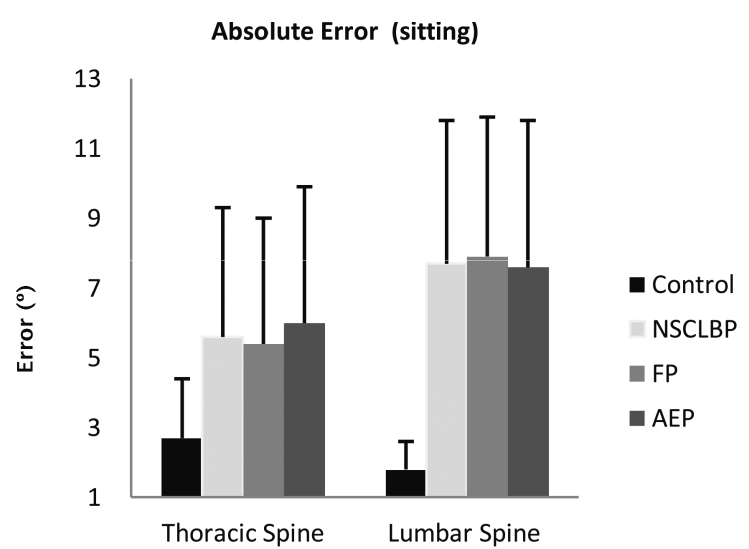

(A)

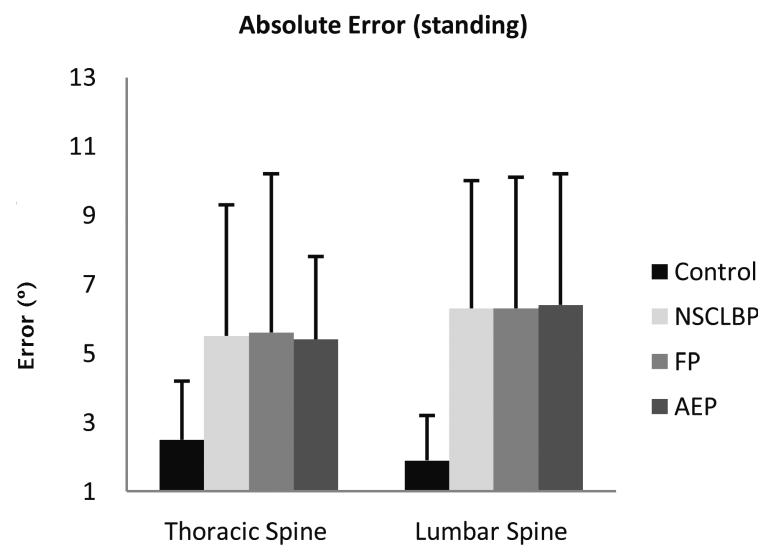

(B)

Figure 1. Absolute error during sitting (A) and standing (B) in asymptomatic controls, NSCLBP (subgroups combined), FP, and AEP subgroups. Error bars represent standard deviation. NSCLBP indicates nonspecific chronic low back pain; FP, flexion pattern; AEP, active extension pattern.

then relaxed into usual sitting for 5 seconds before being instructed to reproduce the target 4 times. In standing, participants first stood in a relaxed position with feet shoulder width apart. They were then assisted in moving through a full spinal flexion and extension 3 times before being positioned by the researcher (LS) into the neutral (midrange) standing position for 5 seconds and instructed to memorize this. Four repositioning tests with 5 seconds of relaxed standing between each trial were then performed. No feedback on the repositioning accuracy was provided. Synchronized recordings of the spinal kinematics and surface electromyogram (sEMG) were collected during each trial.

\section{Spinal Kinematics}

C7, T12, and S1 spinous processes were identified by the researcher (LS), checked by the physiotherapist (VS), and retro-reflective markers were affixed in relaxed sitting to limit the effect of displacement attributed to skin movement. ${ }^{43}$ Thoracic and lumbar spines were measured using a 3-dimensional kinematic motion analysis system (VICON 512, VICON Motion Systems Ltd, Oxford, UK) that detected motion of the 


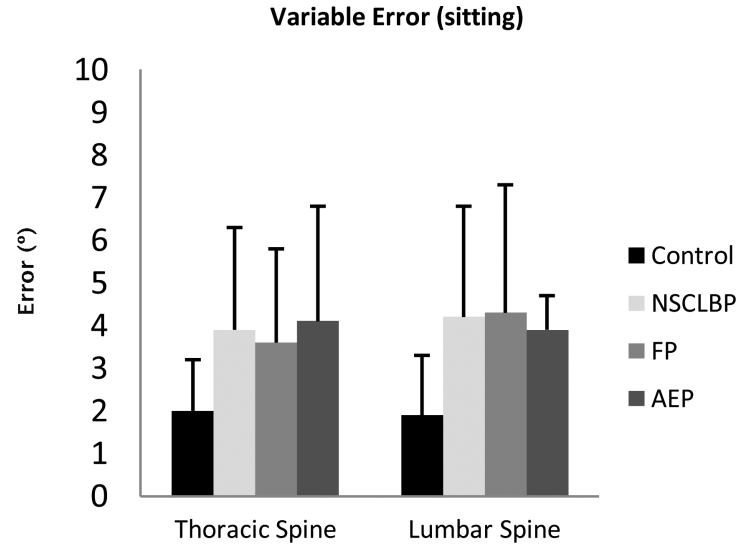

(A)

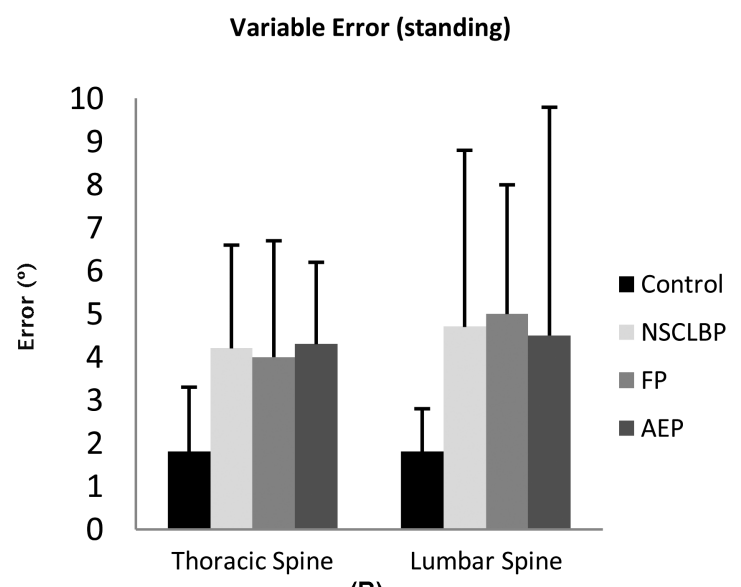

(B)

Figure 2. Variable error during sitting (A) and standing (B) in asymptomatic controls, NSCLBP (subgroups combined), FP, and AEP subgroups. Error bars represent standard deviation. NSCLBP indicates nonspecific chronic low back pain; FP, flexion pattern; AEP, active extension pattern.

Spinal Wheel, a hand-held device with a reflective marker in its center, which was guided along the spinal groove, to obtain the trace of the thoracic and lumbar curvatures. This method demonstrated excellent intratester reliability (intraclass correlation coefficient $=0.947-0.980)$ and acceptable measurement error $\left(1.8^{\circ}-3.7^{\circ}\right) .^{44}$ In Matlab 7.0, the Spinal Wheel curvature was subdivided into 19 equidistant points, and an angle between the lines interconnecting the adjacent points was calculated. Positive values indicated flexion and negative values represented extension. The sum of 11 angles between T1 and T12 and 4 angles between L1 and L5 represented the thoracic and lumbar curvatures, respectively. Target and repositioning data were used to calculate absolute error (AE), representing error magnitude; variable error (VE), representing error consistency; and constant error (CE), representing error direction.

\section{Trunk Muscle Activity}

Eight-channel sEMG (Octopus Cable Telemetric System; Bortec Electronics Inc., Calgary, Alberta, Canada) recorded activity of left (L) and right (R) superficial lumbar multifi-

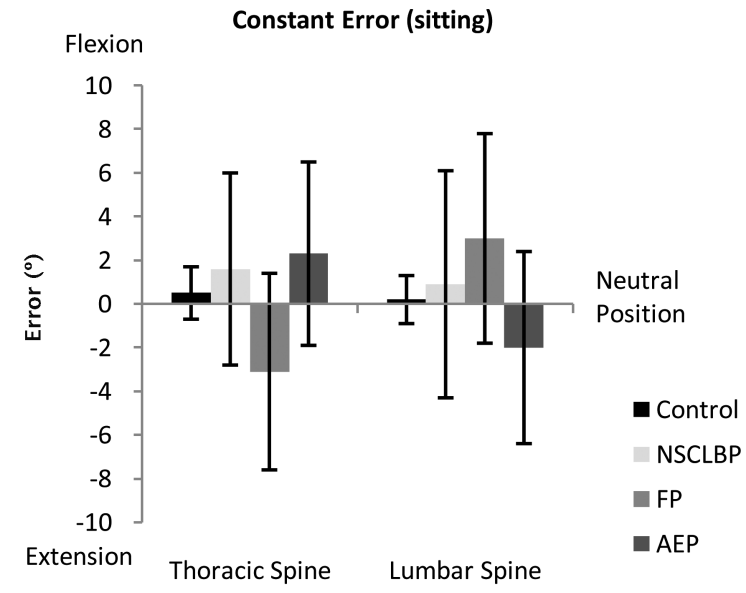

(A)

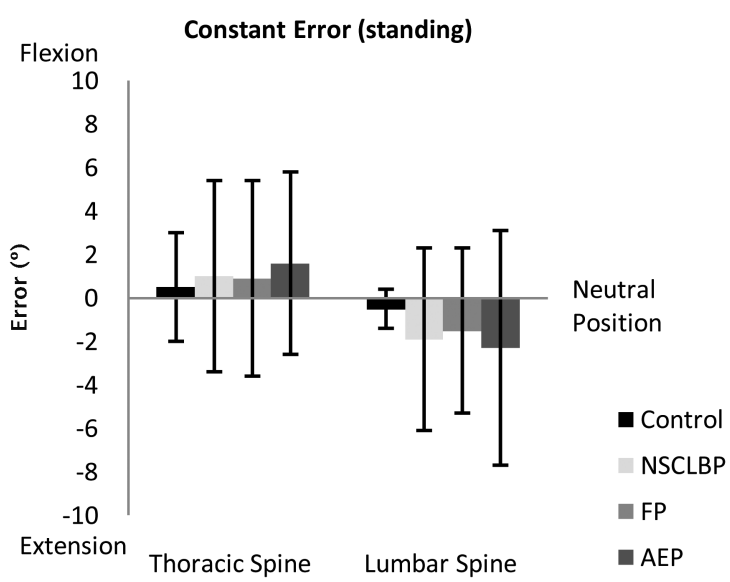

(B)

Figure 3. Constant error during sitting (A) and standing (B) in asymptomatic controls, NSCLBP (subgroups combined), FP, and AEP subgroups. Error bars represent standard deviation. NSCLBP indicates nonspecific chronic low back pain; FP, flexion pattern; AEP, active extension pattern.

dus (LM), iliocostalis lumborum pars thoracic (ICLT), external oblique (EO), and transverse fibers of internal oblique (TrIO). Standard electrode placement and skin preparation procedures were used, as reported elsewhere. ${ }^{45}$ Trunk muscle activity was recorded on completion of each sitting and standing repositioning trial. Raw sEMG signals were preamplified with a fixed gain of 500, a common rejection ratio of 115 $\mathrm{dB}$, and frequency response between 10 and $1000 \mathrm{~Hz}$, visually inspected for heartbeat artifacts, full-wave rectified, and band pass filtered (0 phase lag, $20-\mathrm{Hz}$ cutoff frequency), by using second-order Butterworth filter to generate a linear envelope for each channel. The sEMG amplitude was normalized using standardized movements that have been shown to produce stable submaximal voluntary contractions and excellent within- and between-day reliability in healthy and NSCLBP individuals. $^{45}$

\section{STATISTICAL ANALYSIS}

Group differences for sex were assessed with $\chi^{2}$ test, age, and body mass index (BMI) with 1-way analysis of variance, and independent $t$ tests investigated pain (visual analogue scale) 


\begin{tabular}{|c|c|c|c|c|c|c|c|c|c|c|}
\hline & \multicolumn{6}{|c|}{ Repositioning Errors (SD) $\left(^{\circ}\right)$} & \multicolumn{4}{|c|}{ Trunk Muscle (SD) sEMG (\% Sub-MVC) } \\
\hline & \multicolumn{2}{|c|}{ Absolute Error } & \multicolumn{2}{|c|}{ Variable Error } & \multicolumn{2}{|c|}{ Constant Error* } & \multirow[b]{2}{*}{ LM } & \multirow[b]{2}{*}{ ICLT } & \multirow[b]{2}{*}{ TrIO } & \multirow[b]{2}{*}{ EO } \\
\hline & Th & $\mathbf{L}$ & Th & $\mathbf{L}$ & Th & $\mathbf{L}$ & & & & \\
\hline \multicolumn{11}{|c|}{ Sitting } \\
\hline $\begin{array}{l}\text { Asymptomatic } \\
\text { controls }\end{array}$ & $2.7(1.7)$ & $1.8(0.8)$ & $2.0(1.5)$ & $1.9(1.0)$ & $0.5(2.2)$ & $0.2(1.1)$ & $11.5(7.0)$ & $11.3(6.7)$ & $21.7(10.0)$ & $17.7(9.3)$ \\
\hline NSCLBP & $5.6(3.7)$ & $7.7(4.1)$ & $3.9(2.4)$ & $4.2(2.6)$ & $1.6(6.2)$ & $0.9(7.7)$ & $14.2(8.6)$ & $13.9(7.9)$ & $30.1(15.5)$ & $26.6(12.6)$ \\
\hline $\mathrm{FP}$ & $5.4(3.6)$ & $7.9(4.0)$ & $3.6(2.7)$ & $4.3(3.0)$ & $-3.1(4.9)$ & $3.0(6.9)$ & $15.0(8.5)$ & $14.7(8.4)$ & $31.8(15.2)$ & $27.2(12.2)$ \\
\hline AEP & $6.0(3.9)$ & $7.6(4.2)$ & $4.1(2.2)$ & $3.9(1.8)$ & $2.3(7.2)$ & $-2.0(7.9)$ & $13.1(8.7)$ & $12.9(7.1)$ & $29.8(15.8)$ & 25.7 (13.3) \\
\hline \multicolumn{11}{|c|}{ Standing } \\
\hline $\begin{array}{l}\text { Asymptomatic } \\
\text { controls }\end{array}$ & $2.5(1.7)$ & $1.9(1.3)$ & $1.8(1.2)$ & $1.8(1.4)$ & $0.6(2.5)$ & $-0.5(0.9)$ & $14.6(6.5)$ & $13.2(6.0)$ & $32.8(11.9)$ & $23.0(11.4)$ \\
\hline NSCLBP & $5.5(3.8)$ & 6.3 (3.7) & $4.2(2.4)$ & 4.7 (4.1) & $1.0(4.4)$ & $-1.9(5.2)$ & $18.9(9.8)$ & $14.1(9.7)$ & $40.8(16.4)$ & 32.5 (15.0) \\
\hline $\mathrm{FP}$ & $5.6(4.6)$ & 6.3 (3.8) & $4.0(1.9)$ & $5.0(5.3)$ & $0.9(4.5)$ & -1.5 (5.8) & $20.3(10.3)$ & $15.8(10.1)$ & 41.7 (16.6) & $33.3(15.0)$ \\
\hline AEP & $5.4(2.4)$ & 6.4 (3.8) & $4.3(2.7)$ & 4.5 (3.0) & $1.6(4.2)$ & $-2.3(4.4)$ & $16.9(8.8)$ & $13.0(9.1)$ & $39.3(15.9)$ & $31.5(15.2)$ \\
\hline
\end{tabular}

*Constant error positive value represents an underestimation of the neutral position target (relative flexion) and negative value represents an overestimation of the neutral position target (relative extension).

sEMG indicates surface electromyography; \% sub-MVC, percentage of submaximal voluntary contraction; Th, thoracic spine; L, lumbar spine; LM, superficial lumbar multifidus; ICLT, iliocostalis lumborum pars thoracis; TrIO, transverse fibers of internal oblique; EO, external oblique; NSCLBP, nonspecific chronic low back pain; FP, flexion pattern; AEP, active extension pattern.

and disability (Roland-Morris Disability Questionnaire) differences in NSCLBP subgroups. For the main analysis, independent $t$ tests compared differences between the asymptomatic and NSCLBP (FP and AEP combined) groups. One-way analysis of variance with post hoc Bonferroni (AE, VE, and sEMG) and Games-Howell tests (normally distributed but unequal subgroup variance CE) compared differences among the asymptomatic, FP, and AEP groups. SPSS 14 (IBM, Chicago, IL) was used, with alpha level $(P=0.05)$ and post hoc comparisons $(P=0.016)$ to reflect the multiple comparisons (FP vs. AEP, asymptomatic vs. FP, and asymptomatic $v s$. AEP).

\section{RESULTS}

Five participants did not attend (3 asymptomatic and 2 NSCLBP), leaving $90 \mathrm{NSCLBP}(\mathrm{FP}=51$, AEP $=39)$ and 35 asymptomatic controls for the final analysis. There was no between-group difference in the group characteristics except for BMI $(P=0.03)$, with FP having higher BMI than the asymptomatic group (Table 2). The repositioning error means and standard deviations are presented in Table 3 and Figures 1 to 3 , and the trunk muscle activity is presented in Table 3 and Figure 4. All group comparisons are detailed in Table 4.

\section{Spinal Position Sense}

The NSCLBP (combined and subclassified) group produced significantly greater $\mathrm{AE}$ and VE than the asymptomatic controls in the sitting and standing thoracic and lumbar spines, with no differences between the subgroups (Table 4). No difference was observed between NSCLBP (combined) and asymptomatic controls in thoracic and lumbar CE during sitting and standing. Only when the NSCLBP was subclassified were differences in CE apparent in the thoracic spine (sitting: $P=0.001$ ) and lumbar spine (sitting: $P=0.003$; standing: $P=0.041)$. In sitting, the FP underestimated the lumbar target and overestimated the thoracic target compared with AEP and asymptomatic groups $(P<0.01)$. Conversely, AEP overestimated the lumbar target and underestimated the thoracic target compared with FP $(P<0.016)$, but not reaching statistical significance compared with the asymptomatic group $(P>0.016)$. In standing, the only significant difference was in the lumbar spine where AEP overestimated the target position compared with the asymptomatic group $(P<0.016)$. There was no difference between subgroups in the thoracic spine during standing $(P>0.016)$.

\section{Trunk Muscle Activity}

Paired $t$ test revealed no significant difference between $\mathrm{L}$ and $\mathrm{R}$ trunk muscles; therefore, the corresponding muscle data were averaged for the final analysis. Compared with the asymptomatic controls, the NSCLBP (FP and AEP combined) produced significantly higher TrIO and EO activity and comparable LM and ICLT activity during sitting and standing (Table 4). After subclassification, differences were apparent in LM during standing $(P<0.017)$, where FP produced 


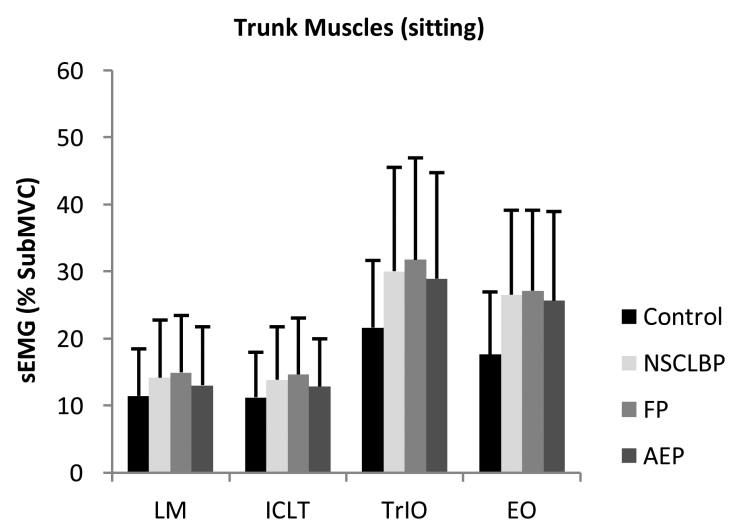

(A)

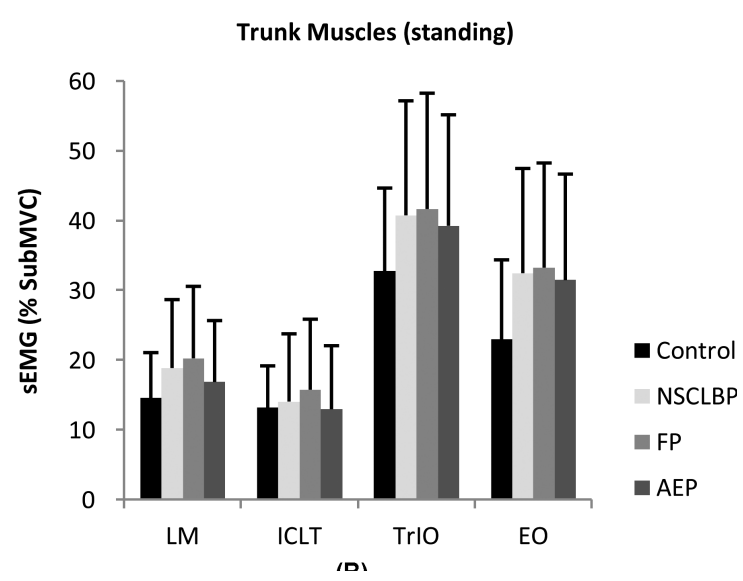

Figure 4. sEMG activity in LM, ICLT, TrIO, and EO during sitting (A) and standing (B) in asymptomatic controls, NSCLBP (subgroups combined), FP, and AEP subgroups. Error bars represent standard deviation. sEMG indicates surface electromyography; sub-MVC, submaximal voluntary contractions; LM, lumbar multifidus; ICLT, iliocostalis lumborum pars thoracis; $\mathrm{TrlO}$, transverse fibers internal oblique; $\mathrm{EO}$, external oblique; NSCLBP, nonspecific chronic low back pain; FP, flexion pattern; AEP, active extension pattern.

higher activity than the asymptomatic controls $(P<0.016)$, although a statistical significance was not reached between FP and AEP subgroups $(P>0.016)$. No subgroup differences were shown in TrIO and EO (sitting and standing), ICLT (sitting and standing), and LM (sitting).

\section{DISCUSSION}

Neutral spine position sense and trunk muscle activity were investigated in subgroups of NSCLBP. Subgroup differences were detected on basis of directional deviations from the neutral target in the thoracic spine (sitting) and the lumbar spine (sitting and standing) but not on the basis of error magnitude and variability. Trunk muscle activity was largely nondiscriminatory between subgroups. In accordance with previous research, ${ }^{15-17}$ the detected subgroup differences in this study were lost when NSCLBP was treated as homogeneous, highlighting the importance of classification. The subgroupspecific directional deviation was reflected in the thoracic and the lumbar spines and was present during sitting in the FP and sitting and standing in the AEP subgroup.

Spine

\section{Flexion Pattern}

FP patients underestimated the lumbar target and overestimated the thoracic target adopting flatter lumbar lordosis and flatter thoracic kyphosis in sitting compared with AEP and asymptomatic controls. Similarly, O'Sullivan et al ${ }^{18}$ demonstrated that patients with flexion-related LBP underestimated the neutral lumbar target compared with controls. Flexed sitting postures have also been demonstrated in FP subgroup of adolescents ${ }^{15}$ and adults. ${ }^{16}$ In this study, the FP subgroup flexion-biased deficit during sitting was not apparent during standing. This may be in keeping with the FP classification being characterized by patients reporting sitting as painprovoking, whereas standing usually eases their pain. ${ }^{41} \mathrm{FP}$ produced significantly higher TrIO and EO (sitting and standing), LM (standing), and similar ICLT (sitting and standing) than the asymptomatic controls. This is in contrast with the study of Dankaerts et al, ${ }^{17}$ in which adults with FP had lower activity in LM, ICLT, and TrIO than AEP and healthy controls, but in agreement with the study of Astfalck et al, ${ }^{15}$ who found no differences in adolescents. Numerous factors affect muscle activity levels. Presence of spinal pain may alter muscle function. ${ }^{46}$ In this study, patients reported mild pain during testing, making the influence of pain on these findings less. The higher BMI in FP subgroup unlikely affected the study results because the higher levels of subcutaneous fat levels would have decreased rather than increased sEMG amplitude. ${ }^{47}$ The functional task evaluated may also influence muscle activity ${ }^{48}$ Unlike during static postures evaluated in the studies discussed earlier, ${ }^{15,17}$ the active adjustment of the lumbar spine in this study may have been associated with substantial recruitment of $\mathrm{LM},{ }^{49}$ particularly as in standing the FP subgroup overestimated the lumbar target.

\section{Extension Pattern}

In contrast with FP, the AEP group overestimated the lumbar target and underestimated the thoracic target, adopting greater lordotic/kyphotic postures than FP. Similarly, compared with FP and controls, postures of greater lordosis were demonstrated previously in AEP adolescents ${ }^{15}$ and adults. ${ }^{16}$ In this study, the AEP group significantly overestimated the standing lumbar target compared with the controls, which may be in line with AEP patients reporting standing as painprovoking. ${ }^{41}$ In this study, muscle activity in AEP was not different to the FP. In contrast, Dankaerts et a ${ }^{17}$ demonstrated that AEP had higher trunk muscle activity than FP and controls, but they were in agreement with Astfalck et $a l,{ }^{15}$ who demonstrated no subgroup differences in adolescents. Clearly, motor patterns in NSCLBP are not yet fully understood and require further research. Moreover, in this study subtle adjustments around the neutral spine position may have led to low sEMG signals, arguably reducing the potential to detect differences. ${ }^{50}$

The FP and AEP subgroups demonstrated direction-specific neutral spine position deficits that coincided with abdominal muscle hyperactivity. It is unclear why the patients present in such a manner. Even small deviations from the neutral spine have been suggested to challenge the load-bearing capacity 
TABLE 4. Results for the Repositioning Errors and sEMG Muscle Activity During Sitting and Standing Asymptomatic/NSCLBP (FP and AEP) Asymptomatic/FP/AEP

\section{Sitting}

\begin{tabular}{|l|l|}
\hline \multicolumn{2}{c}{$t$ test } \\
\hline$t$ &
\end{tabular}

test

\begin{tabular}{l|c|c} 
& \multicolumn{2}{c}{ 1-way ANOVA } \\
\hline $\boldsymbol{P}$ & $\boldsymbol{F}_{2,122}$ & $\boldsymbol{P}$
\end{tabular}

Post hoc Tests

\section{Absolute error}

\begin{tabular}{|c|r|}
\hline Th & \\
\hline $\mathrm{L}$ & 10.4 \\
& \\
\hline
\end{tabular}

4.6

Variable error

\begin{tabular}{|c|c|}
\hline Th & \\
\hline $\mathrm{L}$ & \\
& \\
\hline
\end{tabular}

Constant error

\begin{tabular}{|l|c|c|c|c|c|}
\hline \multicolumn{1}{|c|}{ Th } & 2.1 & 0.070 & 7.023 & $0.001^{*}$ \\
\hline $\mathrm{L}$ & 0.842 & 0.402 & 6.030 & $0.003^{*}$ \\
\hline LM & 1.54 & & & & 0.190 \\
\hline ICLT & 1.31 & 0.126 & 1.686 & 0.355 \\
\hline TrIO & 2.93 & 0.192 & 5.566 & $0.006^{*}$ & \\
\hline EO & 3.54 & $0.004^{*}$ & 6.324 & $0.002^{*}$ & \\
\hline
\end{tabular}

\section{Standing}

Absolute error

\begin{tabular}{|c|c|c|c|c|c|}
\hline \multicolumn{1}{|c|}{ Th } & 4.6 & $0.001^{*}$ & 23.06 & $0.001^{*}$ \\
\hline $\mathrm{L}$ & 10.3 & $0.001^{*}$ & 53.78 & $0.001^{*}$ \\
\hline Variable error & 4.93 & $0.001^{*}$ & 12.182 & $0.001^{*}$ \\
\hline Th & & & & \\
\hline $\mathrm{L}$ & 4.75 & $0.001^{*}$ & 11.850 & $0.001^{*}$ \\
\hline
\end{tabular}

10.4

3.18

5.21

$0.001^{*}$

14.553

$0.001 *$

$0.001 *$

$0.001^{*}$

$0.001^{*}$
AEP vs. H† FP vs. AEP

FP vs. H† AEP vs. H† FP vs. AEP

FP vs. AEPt FP vs. H† AEP vs. $\mathrm{H}$

FP vs. AEPt FP vs. H† AEP vs. H

FP vs. H十 AEP vs. H† AEP vs. FP

FP vs. H† AEP vs. H† AEP vs. FP

\footnotetext{
Copyright ( 2012 Lippincott Williams \& Wilkins. Unauthorized reproduction of this article is prohibited.
} 


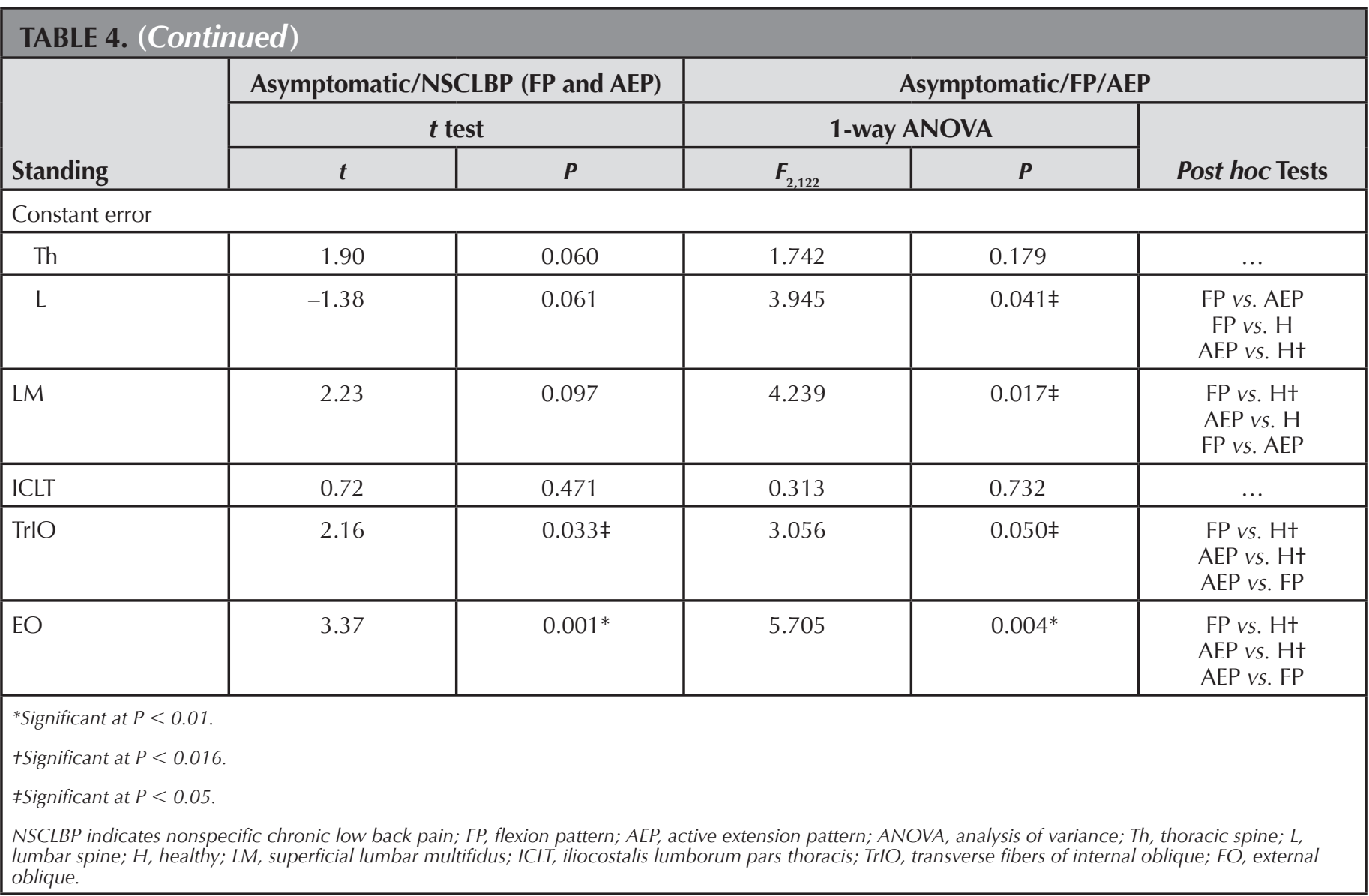

of the spinal tissues, ${ }^{51}$ potentially increasing spinal tissue strain. ${ }^{52}$ Direction-specific loading of already pain-sensitized tissues may exacerbate the disorder, potentially contributing to spinal degeneration. ${ }^{25,41}$ Degeneration may in turn challenge the mechanical integrity of the spine forcing the trunk muscles to adopt stabilizing strategies. ${ }^{52}$ Higher activation of antagonistically acting abdominal muscles in this study has been described previously as a functional adaptation with a purpose of maintaining spinal stability. ${ }^{47}$ Nevertheless, prolonged increase of trunk muscle activity may be metabolically and mechanically costly, ${ }^{53}$ potentially exacerbating LBP. Moreover, FP and AEP patients repositioned into the painprovoking directions. The sensation of pain should arguably reinforce their desire to adopt postures away from the painprovoking movement. This does not seem the case from this study results. It may be hypothesized that the repositioning deficit and abdominal muscle hyperactivity in FP and AEP patients in this study may be maladaptive not allowing them to deviate from the respectively flexed or extended postures, potentially perpetuating the pain disorder. Rehabilitation strategies normalizing these maladaptive patterns may therefore be warranted. ${ }^{41}$

\section{Clinical Implications}

The directional subgroup differences in the neutral spine position in this study may help guide specific interventions. Postural rehabilitation for FP may focus on regaining lumbar

Spine lordosis awareness and control, whereas AEP patients may benefit from regaining movement, awareness, and control in lumbar flexion.

\section{Limitations and Future Research}

Surface EMG has its limitations potentially introducing "cross talk" from the overlying muscles. ${ }^{54}$ Fine-wire EMG would help overcome this and allow evaluation of other muscles, including deep multifidus, suggested to play a role in the control of spinal lordosis. ${ }^{49}$ Investigation of muscle timing and ratios may help explore the complexity of the trunk muscle function in NSCLBP subgroups. The specific directional bias in FP and AEP also needs to be investigated throughout different functional tasks.

\section{CONCLUSION}

Subgroup differences in NSCLBP were detected in some but not all parameters of spinal control. Regardless of classification, both subgroups of NSCLBP demonstrated greater neutral spinal repositioning deficits in error magnitude and variability and higher abdominal activity compared with healthy controls. Back muscle activity was largely nondiscriminatory between the subgroups except for superficial LM. Clear subgroup differences were revealed when considering the direction of the repositioning deficit. These directional differences may guide specific interventions, highlighting the importance and clinical applicability of classifying patients with NSCLBP.

Copyright ( 2012 Lippincott Williams \& Wilkins. Unauthorized reproduction of this article is prohibited. 


\section{Key Points}

$\square$ Spinal position sense and trunk muscle activity when repositioning thoracic and lumbar spine into neutral (midrange) spinal position during sitting and standing were evaluated in subgroups of NSCLBP and asymptomatic controls.

$\square$ Subgroup differences were shown in some but not all parameters.

$\square$ Subgroups of NSCLBP can be detected on the basis of directional deviation of the repositioning error but not on the basis of error magnitude or variability.

$\square$ The subgroup differences in direction of error are apparent in the thoracic spine (sitting) as well as the lumbar spine (sitting and standing).

Although both NSCLBP subgroups had significantly higher abdominal muscle activity compared with asymptomatic controls, the trunk muscle activity between the NSCLBP subgroups was largely nondiscriminatory, with the exception of superficial lumbar multifidus during standing.

\section{Acknowledgments}

SiliconCoach provided financial support for travel and attendance of conferences.

The authors are grateful to the participants who took part in the study. The authors thank Matt Townsend of Cardiff University for technical assistance throughout the study. Many thanks to SiliconCoach for providing conference travel support.

\section{References}

1. Koes BW, van Tulder MW, Thomas S. Diagnosis and treatment of low back pain. BMJ 2006;332:1430-4.

2. Van Tulder MW, Koes BW. Low back pain: acute. Clinical Evidence. London: BMJ Publishing Group; 2006;1633-53.

3. Croft PR, Macfarlane GJ, Papageorgiou AC, et al. Outcome of low back pain in general practice: a prospective study. BMJ 1998;316:1356-9.

4. Waddell G. The Revolution of Back Pained. Edinburgh: Churchill Livingstone; 2004.

5. Burnett AF, Cornelius MW, Dankaerts W, et al. Spinal kinematics and trunk muscle activity in cyclists: a comparison between healthy controls and non-specific chronic low back pain subjects-a pilot investigation. Man Ther 2004;9:211-9.

6. O'Sullivan PB. Clinical instability of the lumbar spine: its pathological basis, diagnosis and conservative management. In: Boyling JD, Jull GA, eds. Grieve's Modern Manual Therapy. Amsterdam: Elsevier; 2004:311-22.

7. Lis AM, Black KM, Korn H, et al. Association between sitting and occupational LBP. Euro Spine J 2007;16:283-98.

8. Adams MA, Mannion AF, Dolan P. Personal risk factors for firsttime low back pain. Spine 1999;24:2497-505.

9. Christie HJ, Kumar S, Warren SA. Postural aberrations in low back pain. Arch Phys Med Rehabil 1995;76:218-24.

10. Mitchell T, O'Sullivan PB, Burnett A, et al. Identification of modifiable personal factors that predict new-onset low back pain: a prospective study of female nursing students. Clin J Pain 2010;26:275-83.

11. Bakker EWP, Verhagen AP, van Trijffel E, et al. Spinal mechanical load as a risk factor for low back pain: a systematic review of prospective cohort studies. Spine 2009;34:E281-93.
12. Fransen MP, Woodward MP, Norton RP, et al. Risk factors associated with the transition from acute to chronic occupational back pain. Spine 2002;27:92-8.

13. Christensen ST, Hartvigsen J. Spinal curves and health: a systematic critical review of the epidemiological literature dealing with associations between sagittal spinal curves and health. I Manipulative Physiol Ther 2008;31:690-714.

14. O'Sullivan PB. Lumbar segmental 'instability': clinical presentation and specific stabilizing exercise management. Man Ther 2000;5: 2-12.

15. Astfalck RG, O'Sullivan PB, Straker LM, et al. Sitting postures and trunk muscle activity in adolescents with and without nonspecific chronic low back pain: an analysis based on subclassification. Spine 2010;35:1387-95.

16. Dankaerts W, O'Sullivan P, Burnett A, et al. Differences in sitting postures are associated with nonspecific chronic low back pain disorders when patients are subclassified. Spine 2006;31:698-704.

17. Dankaerts W, O'Sullivan P, Burnett A, et al. Altered patterns of superficial trunk muscle activation during sitting in nonspecific chronic low back pain patients: importance of subclassification. Spine 2006;31:2017-23.

18. O'Sullivan PB, Burnett A, Floyd AN, et al. Lumbar repositioning deficit in a specific low back pain population. Spine (Phila Pa 1976) 2003;28:1074-9.

19. Geldhof E, De Clercq D, De Bourdeaudhuij I, et al. Classroom postures of 8-12 year old children. Ergonomics 2007;50:1571-81.

20. O'Sullivan PB, Mitchell T, Bulich P, et al. The relationship between posture and back muscle endurance in industrial workers with flexion-related low back pain. Man Ther 2006;11:264-71.

21. Solomonow M, Baratta RV, Zhou BH, et al. Muscular dysfunction elicited by creep of lumbar viscoelastic tissue. J Electromyogr Kinesiol 2003;13:381-96.

22. King K, Davidson B, Zhou BH, et al. High magnitude cyclic load triggers inflammatory response in lumbar ligaments. Clin Biomech $2009 ; 24: 792-8$.

23. Claude LN, Solomonow M, Zhou BH, et al. Neuromuscular dysfunction elicited by cyclic lumbar flexion. Muscle Nerve 2003;27:348-58.

24. Olson MW, Li L, Solomonow M. Flexion-relaxation response to cyclic lumbar flexion. Clin Biomech 2004;19:769-76.

25. Solomonow M. Sensory-motor control of ligaments and associated neuromuscular disorders. I Electromyogr Kinesiol 2006;16: 549-67.

26. Scannell JP, McGill SM. Lumbar posture-should it, and can it, be modified? A study of passive tissue stiffness and lumbar position during activities of daily living. Phys Ther 2003;83: 907-17.

27. Suni J, Rinne M, Natri A, et al. Control of the lumbar neutral zone decreases low back pain and improves self-evaluated work ability: a 12-month randomized controlled study. Spine 2006;31: E611-20.

28. O'Sullivan P. Classification of lumbopelvic pain disorders-why is it essential for management? Man Ther 2006;11:169-70.

29. Magill RA. Motor Learning and Control: Concepts and Applications. 8 ed. New York: McGraw-Hill; 2007.

30. Brumagne S, Cordo P, Lysens R, et al. The role of paraspinal muscle spindles in lumbosacral position sense in individuals with and without low back pain. Spine 2000;25:989-94.

31. Main CJ, Wood PL, Hollis S, et al. The Distress and Risk Assessment Method. A simple patient classification to identify distress and evaluate the risk of poor outcome. Spine 1992;17:42-52.

32. Koumantakis GA, Winstanley J, Oldham JA. Thoracolumbar proprioception in individuals with and without low back pain: intratester reliability, clinical applicability, and validity. J Orthop Sports Phys Ther 2002;32:327-35.

33. Bratcher TL, Moran MA, Zimmer WJ. Tables of sample sizes in the analysis of variance. J Qual Tech 1970;2:156-64.

34. Dworkin SF, Von KorffM, Whitney CW, et al. Measurement of characteristic pain intensity in field research. Pain 1990;41:S290.

35. Roland M, Fairbank J. The Roland-Morris Disability Questionnaire and the Oswestry Disability Questionnaire. Spine 2000;25: 3115-24.

April 2012 
36. Johansson E, Lindberg P. Subacute and chronic low back pain. Reliability and validity of a Swedish version of the Roland and Morris Disability Questionnaire. Scand J Rehabil Med 1998;30:139-43.

37. Jensen MP, Strom SE, Turner JA, et al. Validity of the Sickness Impact Profile Roland scale as a measure of dysfunction in chronic pain patients. Pain 1992;50:157-62.

38. Stratford PW, Binkley JM. A comparison study of the back pain functional scale and Roland Morris Questionnaire. North American Orthopaedic Rehabilitation Research Network. J Rheumatol 2000;27:1928-36.

39. Dankaerts W, O'Sullivan P. The validity of O'Sullivan's classification system for a sub-group of NS-CLBP with motor control impairment (MCI): overview of a series of studies and review of the literature. Man Ther 2011;16:9-14.

40. Elvey RJ, O'Sullivan PB. A contemporary approach to manual therapy. In: Boyling JD, Jull GA, eds. Grieve's Modern Manual Therapy: The Vertebral Column. Amsterdam: Elsevier; 2004:471-93.

41. O'Sullivan P. Diagnosis and classification of chronic low back pain disorders: maladaptive movement and motor control impairments as underlying mechanism. Man Ther 2005;10:242-55.

42. Dankaerts W, O'Sullivan PB, Straker LM, et al. The inter-examiner reliability of a classification method for non-specific chronic low back pain patients with motor control impairment. Man Ther 2006;11:28-39.

43. Kuo Y-L, Tully EA, Galea MP. Skin movement errors in measurement of sagittal lumbar and hip angles in young and elderly subjects. Gait Posture 2008;27:264-70.

44. Sheeran L, Sparkes V, Busse M, et al. Preliminary study: reliability of the spinal wheel. A novel device to measure spinal postures applied to sitting and standing. Eur Spine J 2010;19:995-1003.
45. Dankaerts W, O'Sullivan PB, Burnett AF, et al. Reliability of EMG measurements for trunk muscles during maximal and submaximal voluntary isometric contractions in healthy controls and chronic low back pain patients. J Electromyogr Kinesiol 2004;14: $333-42$.

46. Graven-Nielsen T, Arendt-Nielsen L. Impact of clinical and experimental pain on muscle strength and activity. Curr Rheumatol Rep 2008;10:475-81.

47. van Dieen JH, Cholewicki J, Radebold A. Trunk muscle recruitment patterns in patients with low back pain enhance the stability of the lumbar spine. Spine 2003;28:834-41.

48. Arokoski JP, Valta T, Kankaanpää M, et al. Activation of lumbar paraspinal and abdominal muscles during therapeutic exercises in chronic low back pain patients. Arch Phys Med Rehabil 2004;85:823-32.

49. Bogduk N. Clinical Anatomy of the Lumbar Spine and Sacrum. New York: Churchill Livingstone Inc.; 1997.

50. Reaz MB, Hussain MS, Mohd-Yasin F. Techniques of EMG signal analysis: detection, processing, classification and applications. Biol Proced Online 2006;8:11-35.

51. Kiefer A, Shirazi-Adl A, Parnianpour M. Stability of the human spine in neutral postures. Eur Spine J 1997;6:45-53.

52. Panjabi MM. The stabilizing system of the spine. Part II. Neutral zone and instability hypothesis. J Spinal Disord 1992;5:390-6; discussion 397.

53. Axler CT, McGill SM. Low back loads over a variety of abdominal exercises: searching for the safest abdominal challenge. Med Sci Sports Exerc 1997;29:804-11.

54. De Luca CJ. The surface electromyography in biomechanics. J Appl Biomech 1997;13:135-63. 